\section{Follow Thompson's map to turn biology from a science into a Science}

SIR - Evelyn Fox Keller, in her Essay

"A clash of two cultures" (Nature 445, 603;

2007), argues that biology may not have general laws and that the approach that is natural to physics would probably not work in biology.

In the opening of his seminal 1917 book On Growth and Form, D'Arcy Thompson quoted the eighteenth-century philosopher Immanuel Kant, who lamented that the field of chemistry had not yet embraced a mechanistic and mathematical expression of chemical phenomena. As a result, according to Kant, chemistry at that time was just a science, rather than a Science with a capital S. Despite Kant's view, however, as Thompson emphasizes, a great quantitative revolution proceeded to transform chemistry into a capital-S Science every bit as rigorous as physics. Thompson goes on to argue that biology is poised for just such a quantitative revolution.

Today, Thompson's thesis is being borne out; biology is becoming an increasingly rigorous quantitative Science that is finding more generality with each publication cycle. Most would agree that mathematical theories of quantitative genetics (including the modern synthesis), populations dynamics, organism interactions, epidemiology, ecosystem processes and growth and metabolism have together revolutionized biology, transforming it into a capital-S Science. This quantitative revolution would have been greatly muted, though, had investigators not been compelled, by Thompson's explicit advice, to identify general patterns and laws, to describe these quantitatively and to search for underlying mechanisms.

In this light, Keller's thesis that biology is a series of exceptional cases is a great leap backwards.

Instead of following Keller's philosophy, biology needs to adhere to Thompson's original roadmap and continue its transformation into a rigorous and quantitative Science. It is not fundamentally different from the physical sciences. Keller is correct to note that biology is often unique, but historical contingency does not preclude the existence of general patterns or mechanisms, and has not limited the development of the arguably more rigorous and quantitative fields of astronomy, geology and economics.

Before accepting Keller's thesis, one must first reject the alternative hypothesis that there are general patterns and that these patterns have their basis in equally general mechanisms. For example, Keller dismisses metabolic networks as one possible law-like phenomenom because 'power laws' are not as ubiquitous as first thought. This is conjecture: a quick glance at the biological scaling literature associated with organismal metabolism, life-history, ecology and even evolutionary dynamics reveals an impressive series of general power-law-like behaviours apparently interrelated by a common mechanistic framework based on organismal metabolism. If there are general patterns with equally general mechanisms, then arguably biology has laws. With bioinformatics, large-scale research networks and new computational techniques, we can rapidly identify the existence of general patterns and illuminate the mechanisms that underlie them.

Reasons to follow Thompson's roadmap for biology could not be more urgent. The need to understand and predict the response of the biosphere to climate change, the spread of emerging diseases, the collapse of biological diversity and the need to improve the human condition through medicine and agriculture - all these demand the development of a quantitative, mechanistic and predictive biology. The viewpoint espoused by Keller does not advance the development of biology into a Science. The future of biology belongs to those who follow Thompson's roadmap. Brian J. Enquist, Scott C. Stark Department of Ecology and Evolutionary Biology, University of Arizona, Tucson, Arizona 85721, USA

\section{Authors defend study that shows high Iraqi death toll}

SIR - In our opinion, your News story about our Lancet paper "Death toll in Iraq: survey team takes on new critics" (Nature 446, 6-7; 2007) has confused the matter rather than clarified it. You outline three criticisms of our work: that there was not enough time to have conducted the survey; that the sampling method suffered from a 'main-street bias'; and that the study team fabricated the data (the last being attributed to anonymous "researchers"). These criticisms have been previously addressed, and have little merit.

On the first point, the 1,849 interviews in 49 days described in our study suggest that 38 interviews had to be conducted each day by our eight interviewers. Although introducing themselves and explaining the confidentiality agreement might have taken interviewers several minutes, the five-question interview would take only a couple of minutes for most households that reported no deaths. The idea that eight interviewers could not conduct a total of 38 interviews in a day is not credible.

Second, we dismiss the suggestion that our sampling over-represented main streets, where car bombs are more likely. As stated in our paper (G. Burnham, R. Lafta, S. Doocy and L. Roberts Lancet 368, 1421-1428; 2006), when excluding the statistically outlying cluster of Falluja from the first report, we estimated 98,000 (95\% c.i.: 8,000-194,000) excess deaths versus 112,000 (95\% c.i.: $69,000-155,000)$ over the same period with the second survey. The first survey was done selecting random starting points with a Global Positioning System unit. The second used the random street-selection process, which is being criticized as biased. It rarely occurs in the field that two sampling methods are used allowing for comparison, and here the results are nearly identical. Moreover, there is no plausible mechanism for a significant main-street bias to operate, because only $15 \%$ of all deaths are from car bombs and other ordnance, and because most violent deaths are believed to occur away from the home.

Third, as for the accusation that researchers fabricated the data, we are ready, willing and eager to have an established international authority take a sample of the cluster forms and go to the field with our interviewers to verify the findings. Until that time, the Coalition and Iraqi governments' statements that during the first three years of occupation, Iraq's violent-death rate was lower than those of Russia, Estonia, Latvia, South Africa and Kazakhstan remain an implausible contrast with our findings.

When Nature called one of our study members in Iraq and asked if local officials joined them during the survey, that individual later clarified to Nature by e-mail that 'local officials' did not mean local clinicians and colleagues. This was inaccurately reported in the Nature summary along with a statement by our co-author that interviewers often worked alone. These points were wrongly cited as contradictions between the study team members in your News story.

All reports will eventually have "criticisms that dogged the study", if previously addressed criticisms with so little merit are given a voice in the press.

Les Roberts ${ }^{\star}$, Gilbert Burnham $\uparrow$

*Program on Forced Migration and Health, Mailman School of Public Health, Columbia University, New York, New York 10032, USA †Center for Refugee and Disaster Response, Bloomberg School of Public Health, Johns Hopkins University, Baltimore, Maryland 21205, USA

Nature stands by its version of the events described in the penultimate paragraph of the above Correspondence - Editor, Nature.

Contributions to Correspondence may be submitted to correspondence@nature.com. They should be no longer than $\mathbf{5 0 0}$ words, and ideally shorter. 